\title{
ON THE MONOGENEITY OF A FUNCTION DEFINED BY AN ALGEBRAIC EQUATION
}

\author{
By H. F. BARER.
}

[Read January 11th, 1906._Received February 3rd, 1906.]

1. The present note offers a proof that, if $P_{1}, \ldots, P_{n}$ be (singlevalued) integral functions of a single variable $x$, and the equation

$$
y^{n}+P_{1} y^{n-1}+\ldots+P_{n}=0
$$

have no root in common with an equation of the same form and lower degree, then all the roots are capable of derivation by analytical continuation of any one of them, so that, according to Weierstrass's use of the word, the various roots constitute together a single monogenic function.* The statement implies a certain form for the roots of the equation, the nature of which will appear in the course of the proof; and it is sufficient to exclude common roots of the two equations for every point of any two-dimensional area.

It may well be that a proof of this proposition has been published already ; but, even in the case when $P_{1}, \ldots, P_{n}$ are integral polynomials in $x$, I am not aware, among the various proofs given, of any which seems to have quite the simplicity of this one; in particular, the proof given in Teierstrass's lectures is based upon the theory of rational functions whose poles are all at one placa. The theorem includes clearly the similar result when $P_{1}, \ldots, P_{n}$ are single-valued functions having only poles for finite singularities ; we have only to replace $y$ by $\eta / \mu$, where $\mu$ is a certain polynomial in $x$.

2. We assume, what is a particular case of Weierstrass's implicit-function theorem (Vorbereitungssatz), that, if $f(x, y)$ be a convergent power series in $x$ and $y$, vanishing when $x=0, y=0$, there being, when $x$ alone is put zero, a series remaining beginning with a term in $y^{n}$, then all the roots $y$ of $f(x, y)=0$ which vanish when $x=0$ are given by an equation

$$
\varpi(x, y)=y^{n}+p_{1} y^{n-1}+\ldots+p_{n}=0,
$$

* The theorem is stated and used in a proof that functions of one variable with an algebraical adition theorem ure elliptic functions (or particular cases of such): Proc. Camb. Fhil. Soc., Vol. xur., 1903, p. 236. 
wherein $p_{1}, p_{2}, \ldots, p_{n}$ are power series in $x$ vanishing for $x=0$. A particular case is the result that the $y$-equation

$$
a_{1} x+y+(x, y)_{2}+\ldots=0,
$$

arising when $n=1$, has one, and only one, solution vanishing when $x=0$, expressible in a form

$$
y=-a_{1} x+a_{2} x^{2}+a_{3} x^{3}+\ldots
$$

3. We give also, for the sake of the nature of the proof, a demonstration of the well known form possible for the roots of an equation $\varpi(x, y)=0$ for the neighbourhood of the origin $x=0$. For this there is no loss of generality in assuming that $\varpi(x, y)$ is incapable of being written as a product of factors of the same form (i.e., with converging power series coefficients vanishing for $x=0$ ), since otherwise we could deal with each factor in turn. We have then

$$
\varpi_{y}^{\prime}(x, y)=\partial \varpi(x, y) / \partial y=n y^{n-1}+(n-1) p_{1} y^{n-2}+\ldots+p_{n-1} ;
$$

form the Sylvester $y$-resultant of $\varpi(x, y)$ and $\varpi_{y}^{\prime}(x, y)$, which, being a rational integral polynomial in $p_{1}, \ldots, p_{n}$, is a power series; if this power series vanish identically, $\varpi(x, y)$ and $\varpi_{y}^{\prime}(x, y)$ have a factor

$$
y^{h}+q_{1} y^{h-1}+\ldots+q_{h}
$$

obtainable by the rational method of greatest common divisor, wherein $q_{1}, \ldots, q_{h}$ are rational in $p_{1}, \ldots, p_{n}$, and therefore all of the form $x^{-\lambda} P(x)$, where $P(x)$ is a convergent power series in $x$; as, however, all the roots of $\varpi(x, y)=0$ vanish with $x$, and the roots of $y^{h}+q_{1} y^{h-1}+\ldots+y_{h}=0$ are chosen from those of $\varpi(x, y)=0$, this can only be so if $\lambda=0$ and $P(x)$ vanish with $x$. We have, however, assumed that $\varpi(x, y)$ is not divisible by any factor of the form then arising. The Sylvester $y$-resultant is therefore not identically zero. It vanishes for $x=0$, since both $\varpi(x, y)$ and $\varpi_{y}^{\prime}(x, y)$ vanish for $x=0, y=0$; but a region can be put about $x=0$ within which no other zeros of this resultant are found : this region, taken circular, we call, momentarily, the domain of the origin. If $x_{0}$ be a point within this domain other than the origin, and $y_{0}$ iny one of the corresponding roots of $\varpi\left(x_{0}, y\right)=0$, we have $\varpi_{y}^{\prime}\left(x_{0}, y\right) \neq 0$; put then $x=x_{0}+\xi, y=y_{0}+\eta$ in $\varpi(x, y)$, so obtaining

$$
\frac{\partial \varpi\left(x_{0}, y_{0}\right)}{\partial x_{0}} \xi+\frac{\partial \varpi\left(x_{0}, y_{0}\right)}{\partial y_{0}} \eta+\ldots=0,
$$

and hence, by the assumed theorem of $\S 2$ above, a power series

$$
y=y_{0}+A_{1}\left(x-x_{0}\right)+A_{2}\left(x-x_{0}\right)^{2}+\ldots,
$$


converging in a certain region about $x_{0}$, this representing the only function satisfying $\varpi(x, y)=0$ and reducing to $y_{0}$ when $x=x_{0}$. A precisely similar form is possible for each of the other roots of $\varpi\left(x_{0}, y\right)=0$. Let $r$ be the least of the $n$ radii of convergence of these $n$ series belonging to $x_{0}$. Putting a small circle about the origin and another circle just within the outer circumference of the domain of the origin, and considering the closed annulus so determined, and the value of $r$ for each point $x_{0}$ of this annulus, we say that a real number greater than zero exists such that $r$ is everywhere greater than this real number.

For let the circle about $x_{0}$, of radius $r$, be called the proper region of $x_{0}$; the statement is that the lower limit $\rho$ of the radii of the proper regions, for points $x_{0}$ within the closed annulus, is greater than zero. Let an area contained in the closed annulus be called suitable if it be con. tained in the proper region of some point within or upon the boundary of itself ; the statement will be justified if it can be shown that the closed annulus can be divided into a number of finite areas each of which is suitable. Let the annulus be divided, for example, by means of $n$ concentric circles and $n$ equidistant radii, into equal sub-regions; if all these $n(n+1)$ sub-regions are not suitable, let an unsuitable one be again sub-divided by $n$ concentric eircles and $n$ equidistant radii; and so on continually. The statement is that this subdivision will not need to be continued indefinitely in order that all the sub-regions may be suitable. For an indefinitely continued sequence of regions, each contained in the preceding, and a definite sub-multiple of its area, must have a limiting point, and the proper region of this limiting point will contain all of the sequence of sub-regions which arise beyond a certain stage in the process of subdivision.*

Returning then to the series (A) expressing the root of $\varpi(x, y)=0$, which reduces to $y_{0}$ when $x=x_{0}$, let $x_{1}$ be a point within the closed unnulus spoken of above, and within the circumference of convergence of (A), but at a less distance from it than the lower limit $\rho$ established above for the distance $r$; let $y_{1}$ be the value of the series (A) at $x=x_{1}$; there exists then one root of $\varpi(x, y)=0$, reducing to $y_{1}$ when $x=x_{1}$, expressible by a power series in $x-x_{1}$, converging for $\left|x-x_{1}\right|<\rho$, and therefore forming a continuation of the series (A) beyond the circle of convergence of $(\mathrm{A})$.

It is thus clear that any root $\left(y_{0}\right)$ of $\varpi(x, y)=0$ can be continued

- I have used this phraseology for some time in expounding Goursat's proof of Cauchy's fundamental theorem for complex integrals, and in other of the many applications of tho principle. E.g., in the Proc. Lond. Math. Soc., Vol. 1., 1903, p. 24. The principle is vearly identical with that called in the theory of aggregates the "Heine-Borel Theorem." 
completely round the closed annulus referred to above, back to the neighbourhood of $x_{0}$; it may not, however, after one circuit, resume its value; it may change into another root of $\varpi\left(x_{0}, y\right)=0$; let it resume its original value after $\mu$ circuits. Put then $x=t^{\mu}$, so that the phase of $t$ increases by $2 \pi$ when the phase of $x$ increases by $2 \pi \mu$, and consider, just as w $\theta$ have considered the equation $\varpi(x, y)=0$, the equation $\varpi\left(t^{\mu}, y\right)=0$, and the root of this reducing to $y_{0}$ when $t=t_{0}$, where $t_{0}^{\mu}=x_{0}$. By precisely the above reasoning this root is a single-valued function of $t$ within the annulus, and developable, as a power series in $t-t_{0}$, about any point $t_{0}$. Thus (by Laurent's theorem) it is capable of a representation $\sum_{-\infty}^{\infty} a_{m} t^{n}$, valid for the whole of the annulus. If, however, $M I$ be greater than the modulus of this series for $|t|=R$, we have $\left|a_{-m}\right|<M L R^{m}$; as all the roots of $\varpi(x, y)=0$ vanish for $x=0$, it follows that the negative powers of $t$ (and the zero power) are absent from the series. Consider now the $\mu$ roots of $\varpi(x, y)=0$ given by the series $\sum_{1}^{m} a_{m} t^{m}$ for

$$
t=t, \quad t=t e^{2 \pi i / \mu}, \quad \ldots, \quad t=t e^{[2 \pi i(\mu-1)] \mu} ;
$$

denote them by $y_{1}, \ldots, y_{\mu}$; if $r$ be a positive integer, the sum

$$
y_{1}^{r}+y_{2}^{r}+\ldots+y_{\mu}^{r}
$$

(or, indeed, any rational integral symmetrical polynomial in $y_{1}, \ldots, y_{\mu}$ ) arises as a convergent power series in $t$, which is, in fact, a single-valued function of $x$, and is therefore a converging power series in $x$, manifestly vanishing for $x=0$; thus $y_{1}, \ldots, y_{\mu}$ are the roots of an equation

$$
y^{\mu}+q_{1} y^{\mu-1}+\ldots+q_{\mu}=0,
$$

whose left side therefore divides $\varpi(x, y)$, and is therefore, as we have assumed $\varpi(x, y)$ not to have such factor for $\mu<n$, identical with it. All the roots of $\varpi(x, y)=0$ in the neighbourhood of the origin are thus shewn to be expressible by a single series in $t$, with $x=t^{n}$.

\section{Consider now an expression}

$$
y^{n}+P_{1} y^{n-1}+\ldots+P_{n}
$$

wherein $P_{1}, \ldots, P_{n}$ are (single-valued) integral functions of $x$. We first prove a lemma similar in form to one given by Gauss in the Disquisitiones Arithmetica, that, if a decomposition be possible,

$$
y^{n}+P_{1} y^{n-1}+\ldots+P_{n}=\left(y^{\mu}+H_{1} y^{\mu-1}+\ldots+H_{\mu}\right)\left(y^{\nu}+K_{1} y^{\nu-1}+\ldots+K_{\nu}\right),
$$


in which $H_{1}, \ldots, H_{\mu}, K_{1}, \ldots, K_{\nu}$ are rational functions of integral functions (and therefore are single-valued functions with no finite singularities other than poles), then such a decomposition is possible in which $H_{1}, \ldots, H_{\mu}$, $K_{1}, \ldots, K_{v}$ are integral functions. For the assumed decomposition can be put into the form

$C\left(y^{n}+P_{1} y^{n-1}+\ldots+P_{n}\right)=\left(R y^{\mu}+R_{1} y^{\mu-1}+\ldots+R_{\mu}\right)\left(S y^{\nu}+S_{1} y^{\nu-1}+\ldots+S_{\nu}\right)$,

where $R, R_{1}, \ldots, R_{\mu}, S, S_{1}, \ldots, S_{\nu}$ are integral functions, and $C$ is an integral polynomial. Consider any simple factor of $C$; from $C P_{n}=R_{\mu} S_{\nu}$ it follows that this factor must divide $R_{\mu}$ or $S_{v}$; from

$$
C P_{n-1}=R_{\mu} S_{\nu-1}+S_{\nu} R_{\mu-1}
$$

it follows that, if this factor divide $R_{\mu}$, it must divide $S_{\nu}$ or $R_{\mu-1}$; and so on. Suppose this factor divides

$$
R_{\mu}, R_{\mu-1}, \ldots, R_{\mu-h+1} \quad \text { and } S_{\nu}, S_{\nu-1}, \ldots, S_{\nu-k+1} ;
$$

then compare the coefficients of $y^{h+k}$, giving $C P_{\mu+\nu-h-k}=R_{\mu-h} S_{\nu-k}+$ terms in which the $R$ factor has a suffix greater than $\mu-h$, or the $S$ factor has a suffix greater than $\nu-k$; this factor of $C$ must then divide either $R_{\mu-h}$ or $S_{\nu-k}$; proceeding thus, we see that this factor of $C$ either divides every one of $R, R_{1}, \ldots, R_{\mu}$ or every one of $S, S_{1}, \ldots, S_{\nu}$; it may then be divided out; and so for every factor of $C$. We thus obtain an equation of the form

$$
y^{n}+P_{1} y^{n-1}+\ldots+P_{n}=\left(R y^{\mu}+\ldots+R_{\mu}\right)\left(S y^{\nu}+\ldots+S_{v}\right),
$$

in which every one of $R, \ldots, R_{\mu}, S, \ldots, S_{\nu}$ is an integral function; and $R S=1$ implies that the inverse of each of $R, S$ is an integral function; they may then be divided throughout. We thus obtain an equation of the form first put down, with every one of $H_{1}, \ldots, H_{\mu}, K_{1}, \ldots, K_{\nu}$ an integral function.

5. If now the equation

$$
P(x, y)=y^{n}+P_{1} y^{n-1}+\ldots+P_{n}=0,
$$

wherein $P_{1}, \ldots, P_{n}$ are integral functions, has a root common with an equation of the same form and lower degree

$$
Q(x, y)=y^{h}+Q_{1} y^{h-1}+\ldots+Q_{h}=0,
$$

this common root will satisfy $H(x, y)=0$, where

$$
H(x, y)=y^{\mu}+H_{1} y^{\mu-1}+\ldots+H_{\mu}
$$

is the highest common factor of $P(x, y)$ and $Q(x, y)$, and $H_{1}, \ldots, H_{\mu}$ are rational functions of the integral functions $P_{1}, \ldots, P_{n}, Q_{1}, \ldots, Q_{n}$. The 
existence of such a factor, however, implies another, and an equation

$$
y^{n}+P_{1} y^{n-1}+\ldots+P_{n}=\left(y^{\mu}+H_{1} y^{\mu-1}+\ldots+H_{\mu}\right)\left(y^{\nu}+K_{1} y^{\nu-1}+\ldots+K_{v}\right),
$$

wherein $K_{1}, \ldots, K_{\nu}$ are also rational functions of integral functions. We have shewn that the existence of such an equation requires an equation of the same form in which $H_{1}, \ldots, H_{\mu}, K_{1}, \ldots, K_{v}$ are actually integral functions.

6. We consider then an equation

$$
P(x, y)=y^{n}+P_{1} y^{n-1}+\ldots+P_{n}=0,
$$

$P_{1}, \ldots, P_{n}$ being integral functions, in which the left side is incapable of being written as a product of factors of the same form; it has, therefore, no root common with an equation of the same form and lower degree.

Then the Sylvester $y$-resultant of $P(x, y)=0$ and $\partial P(x, y) / \partial y=0$ does not vanish identically, This resultant is a rational polynomial in the integral functions $P_{1}, \ldots, P_{n}$, and therefore also an integral function; therefore no two of its zeros are within an unassignable nearness of one another, and the number of them within any assigned finite portion of the plane is finite. Taking then any circle not including any zero of this resultant, there exists about any interior point of this circle a power series development for any root of $P(x, y)=0$, just as in $\S 3$, and, as there, this development for any one root can be continued over the whole of this circle. It follows that the root is representable over the whole of the circle by a single development about its centre. Taking next a circle whose centre is at one of the points $x_{0}$ where the resultant vanishes, but not including any other such point, the equations $P\left(x_{0}, y\right)=0, \partial P\left(x_{0}, y\right) / \partial y=0$ have at least one root in common. If $y_{1}$ be a root of the former equation which is not a root of the latter, there exists, as before, a root of $P(x, y)=0$ which reduces to $y_{1}$ when $x=x_{0}$, and is representable as a power series in $x-x_{0}$. If $y_{0}$ be a root of the former equation which is also a root of the latter equation, put $x-x_{0}=\xi, y-y_{0}=\eta$. The roots of $P(x, y)=0$ which reduce to $y_{0}$ when $x=x_{0}$ are then given (by Weierstrass's theorem, $\S 2$ ) by an equation

$$
\varpi(\xi, \eta)=\eta^{\mu}+p_{1} \eta^{\mu-1}+\ldots+p_{\mu}=0,
$$

wherein $p_{1}, \ldots, p_{\mu}$ are power series in $\xi$ vanishing for $\xi=0$, the number $\mu$ being the exponent of the lowest power of $\eta$ in $P\left(x_{0}, y_{0}+\eta\right)$, it being impossible that $y^{n}+P_{1} y^{n-1}+\ldots+P_{n}$ should vanish identically when $x$ is put equal to $x_{0}$; the solutions of the equation $\varpi(\xi, \eta)=0$ are then expressible as before by a set of series of the form $\xi=t^{\lambda}, \eta=P(t)$, there being as many such pairs as irreducible factors of $\varpi(\xi, \eta)$ of the same form. 
7. Taking now any root of $P(x, y)=0$, expressed as a power series in $x-x^{\prime}$, about any point $x^{\prime}$ which is not a zero of the Sylvester $y$-resultant of $P(x, y)$ and $\partial P(x, y) / \partial y$, let it be continued in all possible ways, by paths issuing from and again returning to the neighbourhood of $x^{\prime}$. By reasoning as in $\S 3$, it follows that such continuation is certainly possible over any region lying in the finite part of the plane so long as a point where the resultant vanishes is not included in this region. By what has been seen paths which do not enclose any of these exceptional points will lead back to the same value, but paths enclosing one or more of these may not do so. Notwithstanding that the number of such exceptional points increases indefinitely when the region of the plane considered is taken more and more extensive, the number of roots so obtainable from the original one by all possible continuations cannot, of course, exceed $n$, and must be a definite number, though we cannot experimentally obtain it by exhausting the infinite number of paths necessary to enclose all the exceptional points. Let $y_{1}, \ldots, y_{m}$ be the roots so obtainable, including the original one, and consider the function $y_{1}^{r}+\ldots+y_{n}^{r}$, where $r$ is a positive integer; about every ordinary point $x^{\prime}$ this function is expressible us a power series in $x-x^{\prime}$, and it can be continued over the whole finite plane by any path not passing through one of the exceptional points, and returns thereby always to the same value; about every exceptional point $x^{\prime}$ it is, however, also expressible as a power series in $x-x^{\prime}$, since the possible determinations of any one root about this point enter symmetrically into the formation of the function; it is, moreover, never infinite for any finite value of $x$, since no root of the equation

$$
y+P_{1}+\frac{P_{2}}{y}+\ldots+\frac{P_{n}}{y^{n-1}}=0
$$

can be so infinite. It is thus an integral function of $x$. Therefore $y_{1}, \ldots, y_{m}$ are the roots of an equation

$$
y^{m}+H_{1} y^{m-1}+\ldots+H_{m}=0,
$$

where $H_{1}, \ldots, H_{m}$ are integral functions. Hence, by the hypothesis as to the polynomial $P(x, y)$, it follows that $m=n$, and the equation $P(x, y)=0$ is satisfied by only one monogenic function; as was to be proved.

8. In conclusion, it is to be remarked that, if $P_{1}, \ldots, P_{n}$ be polynomials in $x$, and $P(x, y)$ be incapable of being written as a product of polynomials in $x$ and $y$, that is, be irreducible in the ordinary sense, then it is equally incapable of being written as a product of factors $y^{\mu}+H_{1} y^{\mu-1}+\ldots+H_{\mu}$, $y^{\nu}+K_{1} y^{\nu-1}+\ldots+K_{\nu}$, wherein $H_{1}, \ldots, K_{\nu}$ are integral functions which are 
1906.] Monogeneity of a function defined by an aLgebraic equation. 123

not all polynomials. For, if $\eta=y / x^{r}$, the equation $P(x, y)=0$ can be written

$$
\eta^{n}+\frac{P_{1}}{x^{r}} \eta^{n-1}+\ldots+\frac{P_{n}}{x^{r n}}=0 .
$$

Let $r$ be taken positive and so large that every one of $\frac{P_{1}}{x^{r}}, \frac{P_{2}}{x^{2 r}}, \ldots, \frac{P_{n}}{x^{n r}}$ vanishes when $x$ increases indefinitely, as is possible when $P_{1}, P_{2}, \ldots, P_{n}$ are all polynomials ; then, for every root $y$ of $P(x, y)=0$, the quotient $y / x^{r}$ diminishes indefinitely for $x$ infinite. Thence, if a certain number of these roots be the roots of an equation $y^{\mu}+H_{1} y^{\mu-1}+\ldots+H_{\mu}=0$, wherein $H_{1}, \ldots, H_{\mu}$ are integral functions, these integral functions must all be capable of being reduced, by division by a proper positive power of $x$, to a form in which they vanish for $x$ infinite; so that they must all be polynomials. The theorem proved in $\S 7$ thus shews that an irreducible algebraic equation with polynomial coefficients is satisfied by only one monogenic function of $x$. 